\title{
Cannabinoids and their therapeutic applications in mental disorders
}

\author{
Maria Scherma, PhD; Anna Lisa Muntoni, MD; Gernot Riedel, PhD; \\ Walter Fratta, PhD; Paola Fadda, PhD
}

\begin{abstract}
Mental disorders represent a significant public health burden worldwide due to their high prevalence, chronically disabling nature, and substantial impact on quality of life. Despite growing knowledge of the pathological mechanisms that underlie the development of these disorders, a high percentage of patients do not respond to first-line clinical treatments; thus, there is a strong need for alternative therapeutic approaches. During the past half-century, after the identification of the endocannabinoid system and its role in multiple physiological processes, both natural and synthetic cannabinoids have attracted considerable interest as putative medications in pathological conditions such as, but not exclusive to, mental disorders. Here, we provide a summary of cannabinoid effects in support of possible therapeutic applications for major depression, bipolar disorder, anxiety, posttraumatic stress disorder, and schizophrenia. Considering this evidence, highlighted benefits and risks of cannabinoid use in the management of these illnesses require further experimental study.

Keywords: anxiety; cannabinoid; cannabis; depression; mental disorders; pharmacotherapy; psychosis

\section{Introduction}

Mental disorders, including mood and anxiety disorders, posttraumatic stress disorder (PTSD), and schizophrenia, represent an important public health problem, affecting about $9.6 \%$ to $27.8 \%$ of the general population worldwide. The pathophysiology is complex and not yet completely understood, although genetic, biological, and environmental factors are probably critical for both onset and progression of these disorders. Antidepressants, benzodiazepines, and antipsychotic drugs represent the main pharmacotherapy; however, $40 \%$ to $60 \%$ of patients do not gain total relief from their impairing symptoms with such treatments. Thus, there is a strong need to develop alternative/complementary treatments. There are several lines of evidence suggesting that a promising pharmacotherapy may be based on drugs that target the endocannabinoid system, which in itself appears deregulated in psychiatric patients. This system is recognized as a fundamental modulator of a large variety of physiological processes, including neurodevelopment, emotional states, stress responses, and cognition, so much so that a defect in its signaling might play a role in the pathophysiological underpinnings of mental illnesses.

Cannabis has been used for millennia for therapeutic purposes, and there are several anecdotal reports of its consumption as a form of self-medication for the alleviation of psychiatric symptoms (eg, anxiety, depression, and mania). However, epidemiological studies consistently demonstrated that heavy cannabis use is associated with

Author affiliations: Department of Biomedical Sciences, Division of Neuroscience and Clinical Pharmacology, University of Cagliari, Italy (Maria Scherma, Walter Fratta, Paola Fadda); Neuroscience Institute, National Research Council of Italy (CNR), Cagliari, Italy (Anna Lisa Muntoni, Paola Fadda); Institute of Medical Sciences, University of Aberdeen, Foresterhill, Aberdeen, Scotland, United Kingdom (Gernot Riedel); National Neuroscience Institute, Pisa, Italy (Paola Fadda). Address for correspondence: Prof Paola Fadda, Department of Biomedical Science, University of Cagliari, Cittadella Universitaria S.P.N.8, 09042 Monserrato (Ca), Italy (email: pfadda@unica.it) 


\section{Original article}

Cannabinoids and mental disorders - Fadda et al

occurrence of psychiatric outcomes, especially in people at risk for psychosis or mood disorders. Hence, evidence supporting cannabis-based interventions for the treatment of mental disorders is equivocal, mainly due to the use of extracts from the whole plant. Cannabis contains various phytocannabinoids, among which $\Delta^{9}$-tetrahydrocannabinol (THC) and cannabidiol (CBD) are the major constituents. Both induce their pharmacological effect by interacting with the endocannabinoid system. THC is the main psychoactive ingredient responsible for either psychotic or affective mental health outcomes at higher doses; on the other hand, low doses can attenuate the subjective response to psychosocial stress and act as anxiolytics. In contrast, CBD represents the nonpsychoactive component of the plant that has been suggested to possess antipsychotic, antidepressant, anxiolytic, and procognitive properties. When the effects of THC or CBD were investigated in the management of psychiatric patients, preliminary results suggest that they both may have potential efficacy.

Currently, there are several clinical trials registered on ClinicalTrials.gov, all aimed at evaluating the potential therapeutic effects of cannabis and synthetic cannabinoids on mental disorders. Here, we provide a summary evidencing their possible therapeutic efficacy for the treatment of major depression, bipolar disorder, anxiety, PTSD, and schizophrenia. The term "medical cannabis" here refers to the use of cannabis preparations (raw cannabis, magistral preparations, and standardized cannabis preparations) as medical therapy to treat diseases or alleviate symptoms. "Medicinal cannabinoids," by contrast, refers to medicinal products with marketing authorization: nabilone (synthetic cannabinoid similar to THC), dronabinol (synthetic THC), nabiximols (plant based with approximately equal quantities $\mathrm{THC} / \mathrm{CBD}$ ), and CBD (plant-based).

\section{Major depressive disorder}

Over the years, clinical evidence led to the hypothesis of a link between defects in the endocannabinoid system and depression. Specifically, under basal conditions, serum levels of the two primary endocannabinoids, anandamide
(AEA) and 2-arachidonoylglycerol (2-AG), were found to be reduced in medication-free women diagnosed with major depression, indicating a deficit in peripheral endocannabinoid activity. ${ }^{1,2}$ Furthermore, the decrease in serum levels of 2-AG was strongly correlated with the duration of the depressive episode, whereas serum AEA levels were negatively correlated with anxiety symptoms. On the other hand, moderate exercise prescribed to improve mood states (ie, feelings of depression, total mood disturbance, and state anxiety) increased serum levels of AEA and related fatty acid ethanolamides (ie, oleoylethanolamide) in women with major depression. ${ }^{3}$ Similarly, 2-AG and AEA serum levels were elevated in patients with major depression treated with electroconvulsive therapy relative to baseline. ${ }^{4}$ In human postmortem studies, cannabinoid receptor type $1\left(\mathrm{CB}_{1}\right)$ density and functionality, as well as $\mathrm{CB}_{1}$ messenger RNA (mRNA) levels, were elevated in the dorsolateral prefrontal cortex of suicide victims with major depression..$^{5-7}$ These findings probably represent a compensatory upregulation to counteract endocannabinoid tone lowering in the central nervous system. In contrast, Eggan et $\mathrm{al}^{8}$ did not find any change in $\mathrm{CB}_{1}$-receptor mRNA and protein levels in the same brain areas of depressed individuals who committed suicide. However, the fact that the expression of $\mathrm{CB}_{1}$ receptor in the anterior cingulate cortex was decreased in depressed patients treated with serotonin-selective reuptake inhibitors is consistent with the notion of an enhanced receptor density in drug-naive patients suffering from major depressive episodes. ${ }^{9}$

As for $\mathrm{CB}_{2}$ receptors, no changes were detected in mRNA levels in the dorsolateral prefrontal cortex of suicide victims with major depression. ${ }^{7}$ However, alterations in gene and protein expression of $\mathrm{CB}_{2}$ receptor were found in the dorsolateral prefrontal cortex of suicide victims with no clinical psychiatric history. ${ }^{10}$ Genetic studies found a significant association between polymorphisms (ie, rs 1049353 and rs806368) in the $\mathrm{CB}_{1}$-receptor gene (CNR1), depression susceptibility, and clinical response to antidepressant medications, particularly in female patients with high comorbid anxiety. ${ }^{11,12}$ Some investigations showed that genetic polymorphisms (ie, Q63R and rs2501432) in the $\mathrm{CB}_{2}$ receptor 


\section{Original article}

Cannabinoids and mental disorders - Fadda et al gene (CNR2) seem to be also associated with depression. ${ }^{13,14}$ Finally, the single nucleotide polymorphism rs 324420 of the gene encoding for the fatty acid amide hydrolase $(F A A H)$, the main enzyme responsible for endocannabinoid degradation, appears to be associated with major depression, mostly in adult individuals who have been exposed to childhood trauma. ${ }^{1,11,15}$

In spite of this strong evidence for an involvement of the endocannabinoid system in the pathophysiology of depression, no clinical trials have been registered to evaluate the efficacy of medical cannabis/medicinal cannabinoids for the treatment of major depression. Most of the evidence for an antidepressant effect of medical cannabis comes from case reports and observational studies. For example, in a systematic review, Walsh et $\mathrm{al}^{16}$ identified several crosssectional investigations in which there was clear evidence for an improvement of mood through medical cannabis. This was corroborated by another recent meta-analytic review in which over $35 \%$ of the participants reported self-medication with cannabis to alleviate symptoms of depression. ${ }^{17}$ Accordingly, a large-scale epidemiological study reported that occasional or even daily consumption of cannabis lowers depressive symptoms compared with nonusers. ${ }^{18}$ On the other hand, another recent systematic review and meta-analysis of randomized controlled trials in which medicinal cannabinoids (eg, dronabinol, nabiximols, and nabilone) were used to treat symptoms related to other medical conditions (eg, chronic pain or multiple sclerosis), highlighted no significant effect on the secondary outcome of depression..$^{19}$ Research also showed that the use of medical cannabis to cope with depressive symptoms is associated with cannabis-related problems. For example, Babson et $\mathrm{al}^{20}$ reported that individuals with heightened depression also had heightened cannabis use because of the beneficial effects of cannabis on perceived sleep quality. This suggests that heavy cannabis use may be associated with an increased risk of developing depression as well as with levels of symptom severity. ${ }^{21}$ Accordingly, independent cohort studies investigating cannabinoid effects on depressive symptoms either in psychiatric patients or in the general population, reported that both medicinal and recreational cannabinoids worsened depressive symptoms. ${ }^{22,23}$ From recent research, however, it appears that acute cannabis use induces an immediate positive effect followed by worsening of depressive symptoms after repeated use. ${ }^{24}$ In addition, a population-based longitudinal study suggests that associ- ations between cannabis use and a more severe course of major depression should be ascribed to concomitant sociodemographic and clinical factors rather than to cannabis use itself. ${ }^{25}$ Thus, at present, the evidence for efficacy and safety of medical cannabis or medicinal cannabinoids in major depression is still weak, and additional clinical studies of high methodological quality are needed.

\section{Bipolar disorder}

It is well documented that bipolar disorder is commonly associated with substance use disorders, with cannabis being the most commonly abused drug. ${ }^{26}$ Some bipolar patients report cannabis use to relieve both manic and depressive symptoms and stated that it was more effective than conventional drugs or helped to relieve the side effects of those medications. ${ }^{27}$ Also, medical cannabis was associated with better neurocognitive function in these patients. ${ }^{28}$ As a corollary, smoking cannabis acted to alleviate mood-related symptoms in at least a subset of bipolar patients. ${ }^{29}$ When evaluating the effects on both mood and neuropsychological performance, Sagar et $a \mathrm{l}^{30}$ found that smoking cannabis may result in partial alleviation of clinical symptoms in the short term. However, when two patients diagnosed with bipolar type I disorder were treated with CBD (initial oral dose of $600 \mathrm{mg}$ reaching $1200 \mathrm{mg} /$ day), no effect in attenuating mania was reported. ${ }^{31}$ Moreover, cannabis use might also worsen the occurrence of manic symptoms in those diagnosed with bipolar disorder and might be associated with an increased risk of onset of new manic symptoms. ${ }^{32}$ When looking at the endocannabinoid signaling, a postmortem study revealed no difference in $\mathrm{CB}_{1}$-receptor density in the dorsolateral prefrontal cortex of bipolar patients compared with age-matched controls. ${ }^{7}$ Also, no evidence of any alteration in the expression of $\mathrm{CB}_{1}$ receptor was found in the anterior cingulate cortex of bipolar patients. ${ }^{9}$ On the other hand, the same authors also demonstrated that the administration of a first-generation antipsychotic significantly decreased the expression of $\mathrm{CB}_{1}$ receptor in the anterior cingulate. ${ }^{9}$ When polymorphisms of $C N R 1$, as well as polymorphisms of genes encoding for other components of the endocannabinoid system (eg, N-acyl phosphatidylethanolamine phospholipase D and FAAH), were investigated in bipolar patients also in relation to antipsychotic response, no association was reported. ${ }^{11,33}$ On the contrary, a significant association was found between bipolar disorder and a rs41311993 (524C/A) polymorphism of CNR2. ${ }^{34}$ 


\section{Original article}

Cannabinoids and mental disorders - Fadda et al
Taken together, these observations highlight the need to conduct further studies to better clarify the role of the endocannabinoid system as a possible pharmacological target for the treatment of bipolar disorder. In this regard, a randomized clinical trial has been registered to examine CBD (150 to $300 \mathrm{mg} /$ day for a period of 12 weeks) as adjunctive treatment for bipolar disorder (ClinicalTrials.gov identifier: NCT03310593). ${ }^{35}$ As primary outcome measure, the investigators will evaluate changes in depressive symptoms through the Montgomery-Åsberg Depression Rating Scale. Another trial aimed to evaluate the cognitive and psychophysiological effects of THC (administered through a vaporizer at doses of 2 and $4 \mathrm{mg}$ ) in bipolar disorder has been registered but is not yet recruiting (ClinicalTrials.gov identifier: NCT03206463). ${ }^{36}$ Finally, the effects of dronabinol (one-time oral administration of $5 \mathrm{mg}$ ), as well as of CBD (one-time oral administration of $600 \mathrm{mg}$ ), on cognitive domains relevant to bipolar disorder, eg, arousal, decision making, cognitive control, inhibition, and temporal perception (sense of timing) will be evaluated in adult patients (ClinicalTrials.gov identifier: NCT04231643). ${ }^{37}$ As a secondary end point, this study will determine, for the first time, the effects of acute exposure on the cerebrospinal fluid levels of AEA.

\section{Anxiety disorders}

As with depression, many anecdotal reports imply that patients with anxiety disorders, as well as subjects with high levels of anxiety, use cannabis for the purposes of selftherapy or coping with anxiety. ${ }^{38}$ Moreover, approximately two-thirds of patients diagnosed with anxiety disorders decreased their use of antianxiety medications after substitution with medical cannabis. ${ }^{39}$ A small trial conducted by Crippa et $\mathrm{a}^{40}$ demonstrated that a single dose of CBD (400 $\mathrm{mg}$ ) reliably lowered state anxiety on single-photon emission computed tomography (SPECT) scanning in patients with social anxiety disorder. Similarly, in a double-blind clinical trial on drug-naive subjects with social anxiety disorder, Bergamaschi et $\mathrm{al}^{41}$ showed that an acute dose of CBD $(600 \mathrm{mg})$ was able to reduce subjective anxiety induced by simulated public speaking. The anxiolytic effects of CBD seem to be related to its predominant actions on limbic and paralimbic cortices (amygdala and the hippocampus, as well as the hypothalamus, the left posterior cingulate gyrus, and the left parahippocampal gyrus), which are widely implicated in the pathophysiology of anxiety. ${ }^{42} \mathrm{~A}$ double-blind registered clinical trial is currently recruiting patients with anxiety to assess the effect of $1 \mathrm{~mL}$ of sublingual CBD tincture (10 $\mathrm{mg} / \mathrm{mL} \mathrm{CBD}$ ) administered three times per day (total daily dose of $30 \mathrm{mg}$ ) for 4 weeks (ClinicalTrials.gov identifier: NCT02548559). ${ }^{43}$ Another ongoing multicenter trial will evaluate the efficacy and safety of daily CBD oil capsules ( $200 \mathrm{mg}$ to $800 \mathrm{mg}$ per day) during an 8 -week period on adults diagnosed with generalized anxiety disorder, social anxiety disorder, panic disorder, or agoraphobia (ClinicalTrials.gov identifier: NCT03549819).${ }^{44}$ Efficacy, tolerability, and safety of CBD oil (150 mg to 300 per day) will also be evaluated in a phase 2 randomized placebo-controlled study design in adults with social anxiety disorder (ClinicalTrials.gov identifier: NCT04086342). ${ }^{45}$ A very recently posted pilot trial (ClinicalTrials.gov identifier: NCT04267679) ${ }^{46}$ will investigate the effect of $25 \mathrm{mg}$ full-spectrum CBD soft gel capsules (up to a total dosage of $100 \mathrm{mg}$ per day) for 12 weeks on individuals with diagnosed anxiety, and the varying anxiolytic effects of cannabis (smoked flower, ingested edible) as a function of the CBD to THC ratio is the subject of another pilot study (ClinicalTrials.gov identifier: NCT03491384). ${ }^{47}$ All these clinical studies have been registered recently and are still in their initiation phase (not yet recruiting), and results cannot be expected until 2021 at the earliest.

It is important to emphasize that cannabis consumption affected anxiety-related behaviors in a dose-dependent manner, with low doses being anxiolytic and high doses inducing increased anxiety and panic. ${ }^{48}$ For instance, a recent double-blind, placebo-controlled study with healthy adults showed that a low dose $(7.5 \mathrm{mg})$ of oral THC significantly reduced self-reported subjective distress after a psychosocial stressor, while high doses $(12.5 \mathrm{mg})$ increased negative mood overall. ${ }^{49}$ At specific doses, neuroimaging studies showed that THC could both increase and decrease negative emotional processing in healthy volunteers. ${ }^{50} \mathrm{On}$ the contrary, CBD exerted anxiolytic effects in humans without inducing anxiogenic effects at high doses at baseline. ${ }^{51}$ When coadministered, CBD was able to attenuate the anxiogenic effect of high doses of THC, but this has not been confirmed more recently. ${ }^{52}$

Data underpinning benefits of medicinal cannabinoids on anxiety outcomes were also provided by multiple randomized clinical trials in which they were tested in patients with other medical conditions. ${ }^{19}$ However, these effects have not always been consistent since lack of efficacy also has been reported. ${ }^{19}$ These conflicting results may pertain to the use 


\section{Original article}

Cannabinoids and mental disorders - Fadda et al

of varying doses and differences in THC vs CBD content as well as to modes of administration (eg, vaporization, oromucosal sprays, or sublingual preparations). Finally, significant correlation between cannabis use and increased risk for the onset of anxiety disorders, with greater symptom severity and with poorer treatment outcome, have also been found.$^{53}$ In light of all these results, further clinical studies are needed to better delineate the therapeutic potential of cannabinoids in anxiety disorders.

\section{Posttraumatic stress disorder}

In the last few years, the hypothesis of an involvement of the endocannabinoid system in the pathophysiology of PTSD has become increasingly widespread. For example, a recent $\mathrm{CB}_{1}$-receptor positron-emission tomography imaging study showed increased $\mathrm{CB}_{1}$-receptor expression in the brains of individuals with PTSD compared with controls, an increase that was accompanied by a significant reduction in peripheral AEA levels. ${ }^{54}$ Accordingly, AEA levels exhibited a negative relationship with the degree of intrusive symptoms among individuals with PTSD. ${ }^{55}$ Reduced peripheral AEA levels together with a compensatory upregulation of $\mathrm{CB}_{1}$ receptor suggest a lower endocannabinoid tone in PTSD and warrant the use of both medical cannabis and medicinal cannabinoids as putative treatment options for improving PTSD symptoms. ${ }^{56}$ As reviewed by Hindocha et al, ${ }^{57}$ different cross-sectional studies reported a reduction in PTSD symptomatology in patients assessed on the Clinician-Administered PTSD Scale (CAPS) and using medical cannabis. Also, Roitman et al, ${ }^{58}$ in an open-label study, revealed that, in patients with unremitted PTSD, treatment with sublingual THC (5 mg) twice daily for 3 weeks had beneficial effects on global symptom severity, sleep quality, frequency of nightmares, and PTSD hyperarousal. Moreover, the capacity of nabilone in reducing the intensity of nightmares and improving sleep in patients with PTSD was reported in three different studies. ${ }^{57}$ Finally, a controlled cohort study analyzed retrospective clinical data regarding variable doses of CBD prescribed to 11 psychiatric outpatients diagnosed with PTSD. Ten of these 11 patients reported an overall decrease in PTSD symptom severity. ${ }^{59}$

However, as stated by a commission of the National Academies of Sciences, Engineering and Medicine, the evidence supporting improvement in PTSD symptoms are limited and insufficient. ${ }^{60}$ Randomized clinical trials that have been registered and are recruiting patients with PTSD to test the efficacy of dronabinol (5 and $10 \mathrm{mg}$; ClinicalTrials.gov identifier: NCT03008005) ${ }^{61}$ and CBD (a fixed dose of $300 \mathrm{mg}$ of a hemp-derived formulation of purified 99.9\% CBD oil; ClinicalTrials.gov identifier: NCT04197102) ${ }^{62}$ will provide more evidence on the effect of medicinal cannabinoids in the future. It is also important to point out that 16 US states have listed PTSD as a condition for the use of medical cannabis.

\section{Schizophrenia}

Human studies have provided consistent and compelling evidence for heightened endocannabinoid signaling in schizophrenic patients. Elevated levels of AEA were found in blood and cerebrospinal fluid of schizophrenic patients that inversely correlated with psychotic symptoms and normalized after pharmacological treatment with antipsychotics. $^{63,64}$ The results suggest that increased endocannabinoid levels act in a protective manner against psychosis. ${ }^{64}$ Even though results are mixed, alterations in $\mathrm{CB}_{1}$-receptor density have been detected in postmortem brain tissues of schizophrenic patients specifically in cortical regions that have been linked to schizophrenia, such as dorsolateral prefrontal cortex and anterior cingulate. ${ }^{9,65,66}$ When positron emission tomography (PET) was used to investigate $\mathrm{CB}_{1}$-receptor availability in vivo in schizophrenic patients, the results were also mixed, with increases in the pons, nucleus accumbens, cingulate, and insular cortex and decreases in the amygdala, caudate, posterior cingulate cortex, hippocampus, hypothalamus, and insula. ${ }^{67-69}$ These data invite the interpretation that changes in endocannabinoid signaling in schizophrenia is region-specific and related to the specific symptomatology of the patient. Nevertheless, differences in sample size, as well as in methodology used, could account for the variances obtained between studies. Clinical evidence also supports a role for $\mathrm{CB}_{2}$ receptors with reduced expression in peripheral blood mononuclear cells of treatment-naive schizophrenic patients with first-episode psychosis, as well as in patients after treatment with olanzapine. ${ }^{63,70}$ Finally, genetic polymorphisms in $C N 1 R$ were implicated in susceptibility to schizophrenia, but there was no consistency for any given association. ${ }^{71}$

A meta-analysis conducted on several longitudinal population studies suggests that cannabis use is linked to an increased risk of developing schizophrenia in vulnerable individuals and to precipitate psychotic symptoms in schizophrenic patients. ${ }^{72}$ 


\section{Original article}

Cannabinoids and mental disorders - Fadda et al
Furthermore, schizophrenic patients who consume cannabis have lower AEA levels than patients with no cannabis use, thus hindering the protective effect exercised by this endocannabinoid. ${ }^{73}$ However, the risk enhancement is related to the amount of cannabis used, in particular with the use of high-potency cannabis variants, ie, high THC and low CBD concentrations. ${ }^{74}$ Indeed, smoking cannabis high in CBD content significantly reduced the occurrence and severity of psychotic symptoms. ${ }^{75}$ On the other hand, the high rate of cannabis use in schizophrenic patients was interpreted as an attempt to self-medicate against negative symptoms or to overcome the feeling of depression and anxiety associated with these symptoms. ${ }^{76}$ Accordingly, an open-label case series reported that increasing doses of dronabinol $(2.5,5$, and $10 \mathrm{mg}$ ) administered for 3 weeks were able to improve schizophrenic symptoms in treatment-refractory patients. ${ }^{77}$ In a randomized controlled clinical trial carried out by Leweke et al, ${ }^{78}$ schizophrenic patients undergoing either CBD or amisulpride treatment for 28 days showed significant improvements in their psychotic symptoms, and no difference in clinical efficacy was detected between the treatments. Moreover, the authors revealed a statistically significant association between the increase in AEA levels and the decrease in psychotic symptoms in patients treated with CBD. A beneficial effect of CBD in patients with schizophrenia was recently confirmed in a multicenter randomized controlled trial. ${ }^{79}$ In this study, patients were randomized to receive CBD (1000 mg) or placebo alongside their existing antipsychotic medication. After 6 weeks of treatment, compared with placebo, the CBD cohort presented with lower levels of positive psychotic symptoms (hallucinations, delusions) and showed greater improvement in cognitive performance. However, a randomized placebo-controlled trial in which stable antipsychotic-treated outpatients with schizophrenia received CBD (600 mg/daily) as adjunct over 6 weeks found no improvement in psychotic symptoms. ${ }^{80}$

The mechanisms underlying the association between cannabis and psychosis in terms of both risk for the development of psychosis and self-medication are not yet fully understood. It is well established that the psychotic outcomes of cannabis are mainly mediated by THC, as its administration in healthy individuals elicits both positive and negative symptoms, as well as cognitive deficits in a dose-dependent manner. ${ }^{81}$ Moreover, THC induces a transient exacerbation in psychotic symptoms and cognitive deficits in schizophrenic patients. ${ }^{82}$ Neuroimaging studies showed that THC-induced psychotic symptoms are associated with the altered activity of several brain areas affected by schizophrenia, including the prefrontal cortex, anterior cingulate cortex, amygdala, and ventral striatum. ${ }^{83}$ Unlike THC, CBD received growing attention for its antipsychotic properties and in healthy subjects was able to counteract the psychotic symptoms elicited by THC.$^{84}$ Although the mechanism through which CBD exerts its antipsychotic effects is still to be clarified, the majority of studies focused on its ability to indirectly increase AEA levels by inhibiting its reuptake. ${ }^{78}$

\section{Conclusions and future directions}

Despite the constant rise of global prevalence rates, many of the current therapeutic options for mental illnesses are far from being ideal in terms of both efficacy and adverse effects. In this scenario, there is an increasing demand, worldwide, for using cannabinoids as a treatment for various mental disorders. Their therapeutic potential is suggested by the fact that the endocannabinoid system on which they act is malfunctioning when evaluated in psychiatric patients. This system plays a fundamental role in a large variety of physiological processes, including neurodevelopment, cognition, emotional states, and stress responses. It is therefore not surprising that its dysregulation may contribute to provoke some of the clinical traits present in patients with mental disorders. Thus, targeting the endocannabinoid system may represent an alternative, or additional, effective approach for their treatment. Although most of the studies reviewed in this article highlight an improvement in psychiatric symptoms, the evidence encouraging the use of cannabinoids in mental disorders is still unconvincing. The few clinical studies done so far have limitations both in the number of patients and in the duration of therapy. Moreover, reliable follow-up information is missing. Furthermore, several investigations evaluating the benefits of cannabinoids on psychiatric symptoms in patients with other medical conditions (eg, chronic pain or multiple sclerosis) have produced mixed results. On the other hand, larger longitudinal studies highlighted harmful consequences associated with regular and heavy cannabis use in psychiatric patients. In light of these facts, high-quality clinical trials will be necessary to confirm the efficacy and safety of cannabinoids specifically in patients diagnosed with a specific psychiatric condition, according to the Diagnostic and Statistical Manual of Mental Disorders. It would also be useful for these clinical studies to be integrated with measuring both circulating and 


\section{Original article}

Cannabinoids and mental disorders - Fadda et al

cerebrospinal endocannabinoid levels and/or by examining differences in endocannabinoid signaling between individuals with psychiatric illness and healthy controls, before and after the treatment with cannabinoids.
Acknowledgments/Disclosures: This work was supported in part by funds from the Department of Biomedical Sciences Project (RICDIP_2012_Fratta_01), University of Cagliari. The authors declare no conflict of interest.

\section{References}

1. Hill MN, Miller GE, Ho WS, Gorzalka BB, Hillard CJ. Serum endocannabinoid content is altered in females with depressive disorders: a preliminary report. Pharmacopsychiatry. 2008;41(2):48-53.

2. Hill MN, Miller GE, Carrier EJ, Gorzalka BB, Hillard CJ. Circulating endocannabinoids and $\mathrm{N}$-acyl ethanolamines are differentially regulated in major depression and following exposure to social stress. Psychoneuroendocrinology. 2009;34(8):1257-1262. 3. Meyer JD, Crombie KM, Cook DB, Hillard CJ, Koltyn KF. Serum endocannabinoid and mood changes after exercise in major depressive disorder. Med Sci Sports Exerc. 2019;51(9):1909-1917.

4. Kranaster L, Hoyer C, Aksay SS, et al. Electroconvulsive therapy enhances endocannabinoids in the cerebrospinal fluid of patients with major depression: a preliminary prospective study. Eur Arch Psychiatry Clin Neurosci. 2017;267(8):781-786.

5. Hungund BL, Vinod KY, Kassir SA, et al. Upregulation of $\mathrm{CB}$ receptors and agonist-stimulated $\left[{ }^{35} \mathrm{~S}\right]$ GTPgS binding in the prefrontal cortex of depressed suicide victims. Mol Psychiatry. 2004;9(2):184-190. 6. Mato S, Pilar-Cuéllar F, Valdizán EM, et al. Selective up-regulation of cannabinoid $\mathrm{CB}_{1}$ receptor coupling to Go-proteins in suicide victims with mood disorders. Biochem Pharmacol. 2018;157:258-265. 7. Choi K, Le T, McGuire J, et al. Expression pattern of the cannabinoid receptor genes in the frontal cortex of mood disorder patients and mice selectively bred for high and low fear. $J$ Psychiatr Res. 2012;46(7):882-889.

8. Eggan SM, Stoyak SR, Verrico CD, Lewis DA. Cannabinoid CB1 receptor immunoreactivity in the prefrontal cortex: comparison of schizophrenia and major depressive disorder. Neuropsychopharmacology. 2010;35(10):2060-2071.

9. Koethe D, Llenos IC, Dulay JR, et al. Expression of $\mathrm{CB}_{1}$ cannabinoid receptor in the anterior cingulate cortex in schizophrenia, bipolar disorder, and major depression. $J$ Neural Transm (Vienna). 2007;114(8):1055-1063.

10. García-Gutiérrez MS, Navarrete F, Navarro G, et al. Alterations in gene and protein expression of cannabinoid $\mathrm{CB}_{2}$ and GPR55 receptors in the dorsolateral prefrontal cortex of suicide victims. Neurotherapeutics. 2018;15(3):796-806.

11. Monteleone P, Bifulco M, Maina G, et al. Investigation of CNR1 and FAAH endocannabinoid gene polymorphisms in bipolar disorder and major depression. Pharmacol Res. 2010;61(5):400-404.

12. Mitjans M, Serretti A, Fabbri C, et al. Screening genetic variability at the CNRI gene in both major depression etiology and clinical response to citalopram treatment. Psychopharmacology. 2013;227(3):509-519.

13. Onaivi ES, Ishiguro H, Gong JP, et al. Functional expression of brain neuronal $\mathrm{CB} 2$ cannabinoid receptors are involved in the effects of drugs of abuse and in depression. Ann N Y Acad Sci. 2008;1139: 434-449.

14. Kong X, Miao Q, Lu X, et al. The association of endocannabinoid receptor genes (CNR1 and CNR2) polymorphisms with depression: a meta-analysis. Medicine (Baltimore). 2019;98(46):e17403.

15. Lazary J, Eszlari N, Juhasz G, Bagdy G. Genetically reduced FAAH activity may be a risk for the development of anxiety and depression in persons with repetitive childhood trauma. Eur Neuropsychopharmacol. 2016;26(6):1020-1028.

16. Walsh Z, Gonzalez R, Crosby K, S Thiessen M, Carroll C, Bonn-Miller MO. Medical cannabis and mental health: a guided systematic review. Clin Psychol Rev. 2017;51:15-29.

17. Kosiba JD, Maisto SA, Ditre JW. Patient-reported use of medical cannabis for pain, anxiety, and depression symptoms: systematic review and meta-analysis. Soc Sci Med. 2019;233:181-192.

18. Denson TF, Earleywine M. Decreased depression in marijuana users. Addict Behav. 2006;31:738-742. 19. Black N, Stockings E, Campbell G, et al. Cannabinoids for the treatment of mental disorders and symptoms of mental disorders: a systematic review and meta-analysis. Lancet Psychiatry. 2019; 6(12):995-1010.

20. Babson KA, Boden MT, Bonn-Miller MO. Sleep quality moderates the relation between depression symptoms and problematic cannabis use among medical cannabis users. Am J Drug Alcohol Abuse. 2013;39:211-216.

21. Mammen G, Rueda S, Roerecke M, Bonato S, Lev-Ran S, Rehm J. Association of cannabis with long-term clinical symptoms in anxiety and mood disorders: a systematic review of prospective studies. J Clin Psychiatry. 2018;79(4).pii:17r11839.

22. Anand D, Paquette C, Bartuska A, Daughters SB. Substance type moderates the longitudinal association between depression and substance use from pre-treatment through a 1-year follow-up. Drug Alcohol Depend. 2019;197:87-94.

23. Bahorik AL, Sterling SA, Campbell CI, Weisner C, Ramo D, Satre DD. Medical and non-medical marijuana use in depression: longitudinal associations with suicidal ideation, everyday functioning, and psychiatry service utilization. $J$ Affect Disord. 2018;241:8-14.

24. Cuttler C, Spradlin A, McLaughlin RJ. A natu- ralistic examination of the perceived effects of cannabis on negative affect. $J$ Affect Disord. 2018;235: 198-205.

25. Feingold D, Rehm J, Lev-Ran S. Cannabis use and the course and outcome of major depressive disorder: a population based longitudinal study. Psychiatry Res. 2017;251:225-234.

26. Pinto JV, Medeiros LS, Santana da Rosa G, et al. The prevalence and clinical correlates of cannabis use and cannabis use disorder among patients with bipolar disorder: a systematic review with meta-analysis and meta-regression. Neurosci Biobehav Rev. 2019; 101:78-84

27. Grinspoon L, Bakalar JB. The use of cannabis as a mood stabilizer in bipolar disorder: anecdotal evidence and the need for clinical research. J Psychoactive Drugs. 1998;30:171-177.

28. Ringen PA, Vaskinn A, Sundet K, et al. Opposite relationships between cannabis use and neurocognitive functioning in bipolar disorder and schizophrenia. Psychol Med. 2010;40(8):1337-13347.

29. Gruber SA, Sagar KA, Dahlgren MK, Olson DP, Centorrino F, Lukas SE. Marijuana impacts mood in bipolar disorder: a pilot study. Ment Health Subst Use. 2012;5:228-239.

30. Sagar KA, Dahlgren MK, Racine MT, Dreman MW, Olson DP, Gruber SA. Joint effects: a pilot investigation of the impact of bipolar disorder and marijuana use on cognitive function and mood. PLoS One. 2016;11(6): $\mathrm{e} 0157060$.

31. Zuardi A, Crippa J, Dursun S, et al. Cannabidiol was ineffective for manic episode of bipolar affective disorder. J Psychopharmacol. 2010;24(1):135-137. 32. Gibbs M, Winsper C, Marwaha S, Gilbert E, Broome M, Singh SP. Cannabis use and mania symptoms: a systematic review and meta-analysis. J Affect Disord. 2015;171:39-47.

33. Pisanu C, Congiu D, Costa $M$, et al. No association of endocannabinoid genes with bipolar disorder or lithium response in a Sardinian sample. Psychiatry Res. 2013;210(3):887-890.

34. Minocci D, Massei J, Martino A, et al. Genetic association between bipolar disorder and $524 \mathrm{~A}>\mathrm{C}$ (Leu133Ile) polymorphism of CNR2 gene, encoding for CB2 cannabinoid receptor. $J$ Affect Disord. 2011;134(1-3):427-430.

35. NIH US National Library of Medicine. Cannabidiol as an Adjunctive Treatment for Bipolar Depression (CBDBD). Available at: https://clinicaltrials. gov/ct2/show/NCT03310593.

36. NIH US National Library of Medicine. Cognitive and Psychophysiological Effects of Delta-9-Tetrahydrocannabinol in Bipolar Disorder (THC-BD). 


\section{Original article}

Cannabinoids and mental disorders - Fadda et al

Available at: https://clinicaltrials.gov/ct2/show/ NCT03206463.

37. NIH US National Library of Medicine. Effects of Cannabis on Cognition and Endocannabinoid Levels in Bipolar Disorder Patients and Healthy Volunteers. Available at: https://clinicaltrials.gov/ ct2/show/NCT04231643.

38. Turna J, Simpson W, Patterson B, Lucas P, Van Ameringen M. Cannabis use behaviors and prevalence of anxiety and depressive symptoms in a cohort of Canadian medicinal cannabis users. $J$ Psychiatr Res. 2019;111:134-139.

39. Piper BJ, DeKeuster RM, Beals ML, et al. Substitution of medical cannabis for pharmaceutical agents for pain, anxiety, and sleep. J Psychopharmacol. 2017;31(5):569-575.

40. Crippa JA, Derenusson GN, Ferrari TB, et al Neural basis of anxiolytic effects of cannabidiol (CBD) in generalized social anxiety disorder: a preliminary report. J Psychopharmacol Oxf Engl. 2011:25:121-130.

41. Bergamaschi MM, Queiroz RH, Chagas MH, et al. Cannabidiol reduces the anxiety induced by simulated public speaking in treatment-naïve social phobia patients. Neuropsychopharmacol. 2011; 36:1219-1226.

42. Crippa JA, Zuardi AW, Garrido GE, et al. Effects of cannabidiol (CBD) on regional cerebral blood flow. Neuropsychopharmacology. 2004;29(2): 417-426.

43. NIH US National Library of Medicine. Sublingual Cannabidiol for Anxiety. Available at: https:// clinicaltrials.gov/ct2/show/NCT02548559. Accessed July 2020.

44. NIH US National Library of Medicine. Cannabidiol or the Treatment of Anxiety Disorders: An 8-Week Pilot Study. Available at: https://clinicaltrials.gov/ct2/show/NCT03549819. Accessed July 2020.

45. NIH US National Library of Medicine. CHI902 for Treatment of Social Anxiety Disorder. Available at: https://clinicaltrials.gov/ct2/show/ NCT04086342. Accessed July 2020.

46. NIH US National Library of Medicine. Cannabidiol for Anxiety. Available at: https://clinicaltrials. gov/ct2/show/NCT04267679. Accessed July 2020. 47. NIH US National Library of Medicine. Anxiety, Inflammation, and Stress. Available at: https://clinicaltrials.gov/ct2/show/NCT03491384. Accessed July 2020.

48. Viveros MP, Marco EM, File SE. Endocannabinoid system and stress and anxiety responses. Pharmacol Biochem Behav. 2005;81:331-342. 49. Childs E, Lutz JA, de Wit $H$. Dose-related effects of delta-9-THC on emotional responses to acute psychosocial stress. Drug Alcohol Depend. 2017; 177:136-144

50. Bossong MG, van Hell HH, Jager G, Kahn RS, Ramsey NF, Jansma JM. The endocannabinoid system and emotional processing: a pharmacological fMRI study with $\Delta^{9}$-tetrahydrocannabinol. Eur Neuropsychopharmacol. 2013;23:1687-1697.
51. Blessing EM, Steenkamp MM, Manzanares J, Marmar CR. Cannabidiol as a potential treatment for anxiety disorders. Neurother J Am Soc Exp Neurother. 2015;12:825-836.

52. Zuardi AW, Shirakawa I, Finkelfarb E, Karniol IG. Action of cannabidiol on the anxiety and other effects produced by delta 9-THC in normal subjects. Psychopharmacology (Berl). 1982;76:245-250.

53. Feingold D, Rehm J, Factor $H$, Redler A, LevRan S. Clinical and functional outcomes of cannabis use among individuals with anxiety disorders: a 3-year population-based longitudinal study. Depress Anxiety. 2018;35(6):490-501.

54. Neumeister A, Normandin MD, Pietrzak RH, et al. Elevated brain cannabinoid $\mathrm{CB}$, receptor availability in post-traumatic stress disorder: a positron emission tomography study. Mol Psychiatry. 2013;18(9):1034-1040.

55. Hill MN, Bierer LM, Makotkine I, et al. Reductions in circulating endocannabinoid levels in individuals with post-traumatic stress disorder following exposure to the World Trade Center attacks. Psychoneuroendocrinology. 2013;38:2952-2961.

56. Orsolini L, Chiappini S, Volpe U, et al. Use of medicinal cannabis and synthetic cannabinoids in post-traumatic stress disorder (PTSD): a systematic review. Medicina (Kaunas). 2019;55(9):pii: E525.

57. Hindocha C, Cousijn J, Rall M, Bloomfield MAP. The effectiveness of cannabinoids in the treatment of posttraumatic stress disorder (PTSD): a systematic review. J Dual Diagn. 2020;16(1):120-139.

58. Roitman P, Mechoulam R, Cooper-Kazaz R, Shalev A. Preliminary, open-label, pilot study of add-on oral $\Delta^{9}$-tetrahydrocannabinol in chronic post-traumatic stress disorder. Clin Drug Investig. 2014:34:587-591.

59. Elms L, Shannon S, Hughes S, Lewis N. Cannabidiol in the treatment of post-traumatic stress disorder: a case series. J Altern Complement Med. 2019;25:392-397.

60. Abrams DI. The therapeutic effects of Cannabis and cannabinoids: an update from the National Academies of Sciences, Engineering and Medicine report. Eur J Intern Med. 2018;49:7-11.

61. NIH US National Library of Medicine. Effects of Delta-9 Tetrahydrocannabinol (THC) on Retention of Memory for Fear Extinction Learning in PTSD: R61 Study. Available at: https://clinicaltrials.gov/ ct2/show/NCT03008005. Accessed July 2020. 62. NIH US National Library of Medicine. Use of CBD Oil in the Treatment of Posttraumatic Stress Disorder. Available at: https://clinicaltrials.gov/ct2/ show/NCT04197102.

63. De Marchi N, De Petrocellis L, Orlando P, Daniele F, Fezza F, Di Marzo V. Endocannabinoid signalling in the blood of patients with schizophrenia. Lipids Health Dis. 2003;2:5.

64. Giuffrida A, Leweke FM, Gerth CW, et al. Cerebrospinal anandamide levels are elevated in acute schizophrenia and are inversely correlated with psychotic symptoms. Neuropsychopharmacol. 2004; 29:2108-2114.
65. Newell KA, Deng C, Huang XF. Increased cannabinoid receptor density in the posterior cingulate cortex in schizophrenia. Exp Brain Res. 2006;172:556-560.

66. Eggan SM, Hashimoto T, Lewis DA. Reduced cortical cannabinoid 1 receptor messenger RNA and protein expression in schizophrenia. Arch Gen Psychiatry. 2008;65:772-784.

67. Wong DF, Kuwabara H, Horti AG, et al. Quantification of cerebral cannabinoid receptors subtype 1 (CB1) in healthy subjects and schizophrenia by the novel PET radioligand $\left[{ }^{11} \mathrm{C}\right] \mathrm{OMAR}$. NeuroImage. 2010;52:1505-1513.

68. Ceccarini J, De Hert M, Van Winkel R, et al. Increased ventral striatal $\mathrm{CB}$, receptor binding is related to negative symptoms in drug-free patients with schizophrenia. NeuroImage. 2013;79:304-312. 69. Ranganathan M, Cortes-Briones J, Radhakrishnan $\mathrm{R}$, et al. Reduced brain cannabinoid receptor availability in schizophrenia. Biol Psychiatry. 2016; 79(12):997-1005.

70. Bioque M, García-Bueno B, Macdowell KS, et al. Peripheral endocannabinoid system dysregulation in first-episode psychosis. Neuropsychopharmacology. 2013;38:2568-2577.

71. Gouvêa ES, Santos AF Filho, Ota VK, et al. The role of the CNR1 gene in schizophrenia: a systematic review including unpublished data. Braz J Psychiatry. 2017;39(2):160-171.

72. Hasan A, von Keller R, Friemel CM, et al. Cannabis use and psychosis: a review of reviews. Eur Arch Psychiatry Clin Neurosci. 2020;270(4): 403-412.

73. Leweke FM, Giuffrida A, Koethe D, et al. Anandamide levels in cerebrospinal fluid of first-episode schizophrenic patients: impact of cannabis use. Schizophr Res. 2007;94:29-36.

74. Di Forti M, Morgan C, Dazzan P, et al. High-potency cannabis and the risk of psychosis. Br J Psychiatry. 2009;195(6):488-491.

75. Schubart CD, Sommer IE, van Gastel WA, Goetgebuer RL, Kahn RS, Boks MP. Cannabis with high cannabidiol content is associated with fewer psychotic experiences. Schizophr Res. 2011;130: 216-221.

76. Mané A, Fernández-Expósito $M$, Bergé $\mathrm{D}$, et al. Relationship between cannabis and psychosis: reasons for use and associated clinical variables. Psychiatry Res. 2015;229(1-2):70-74.

77. Schwarcz G, Karajgi B, McCarthy R. Synthetic delta-9-tetrahydrocannabinol (dronabinol) can improve the symptoms of schizophrenia. J Clin Psychopharmacol. 2009;29:255-258.

78. Leweke FM, Piomelli D, Pahlisch F, et al. Cannabidiol enhances anandamide signaling and alleviates psychotic symptoms of schizophrenia. Transl Psychiatry. 2012;2:e94.

79. McGuire P, Robson P, Cubala WJ, et al. Cannabidiol (CBD) as an adjunctive therapy in schizophrenia: a multicenter randomized controlled trial. Am J Psychiatry. 2018;175(3): 225-231. 


\section{Original article}

Cannabinoids and mental disorders - Fadda et al

80. Boggs DL, Surti T, Gupta A, et al. The effects of cannabidiol (CBD) on cognition and symptoms in outpatients with chronic schizophrenia a randomized placebo controlled trial. Psychopharmacology (Berl). 2018;235(7):1923-1932.

81. Morrison PD, Zois V, McKeown DA, et al. The acute effects of synthetic intravenous $\Delta^{9}$-tetrahydrocannabinol on psychosis, mood and cognitive functioning. Psychol Med. 2009;39(10):1607-1616.
82. D'Souza DC, Abi-Saab WM, Madonick S, 84. Bhattacharyya S, Morrison PD, Fusar-Poli P, et al. Delta-9-tetrahydrocannabinol effects in et al. Opposite effects of $\Delta^{9}$-tetrahydrocannabinol schizophrenia: implications for cognition, psycho- and cannabidiol on human brain function and sis, and addiction. Biol Psychiatry. 2005;57(6): psychopathology. Neuropsychopharmacology. 594-608.

83. Murray RM, Englund A, Abi-Dargham A, et al. Cannabis-associated psychosis: neural substrate and clinical impact. Neuropharmacology. 2017;124: 89-104. 
